\title{
The Influence of Fast Neutron Irradiation on the Magnetostriction of Ceramic $\mathrm{YBa}_{2} \mathrm{Cu}_{3} \mathrm{O}_{7-\delta}$ Sample
}

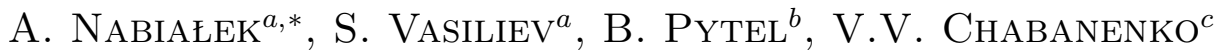 \\ AND S. PIECHOTA ${ }^{a}$ \\ ${ }^{a}$ Institute of Physics, Polish Academy of Sciences \\ al. Lotników 32/46, 02-668 Warsaw, Poland \\ ${ }^{b}$ Institute of Atomic Energy, 05-400 Otwock-Świerk, Poland \\ ${ }^{c}$ Donetsk Physico-Technical Institute, Ukrainian Academy of Sciences \\ 72 R. Luxemburg str., 83114, Donetsk, Ukraine
}

We investigated the magnetostriction of ceramic $\mathrm{YBa}_{2} \mathrm{Cu}_{3} \mathrm{O}_{7-\delta}$ sample both before and after fast neutron irradiation with the fluency of $7.55 \times 10^{17} \mathrm{n} / \mathrm{cm}^{2}$ and energy of above $0.5 \mathrm{MeV}$. We have found that after irradiation the widths of both transverse and longitudinal magnetostriction hysteresis loops at a given temperature and at a given external magnetic field increased several times. At $4.2 \mathrm{~K}$ and in 10 tesla the widths of the magnetostriction hysteresis loop of the irradiated sample are of an order of $10^{-5}$. The results can be understood in the framework of the model of the magnetostriction induced by the pinning forces, assuming the stress in the sample to be induced mainly by the intragrain screening currents.

PACS numbers: 74.25.Qt, 74.72.Bk, 75.80.+q

\section{Introduction}

In high temperature superconductors (HTSs) the experimentally observed phenomenon of magnetostriction is predominated by the mechanism of the magnetostriction induced by the pinning forces [1]. In large crystals of these compounds giant magnetostriction, of an order of $10^{-4}$ (the number means relative changes of the sample dimensions), at $4.2 \mathrm{~K}$ and in an external magnetic field of several tesla

*corresponding author; e-mail: nabia@ifpan.edu.pl 
was found [1, 2]. As in the case of the well-known Bean model [3], assuming the critical current density to be independent (or at least weakly dependent) on the magnetic field, it is easy to correlate the critical current density with the width of the magnetization hysteresis loop, a similar procedure can also be applied in the case of the magnetostriction hysteresis loops. For an isotropic material and for some simple sample geometries (slab or cylinder) the width of the transverse magnetostriction hysteresis loop $\left(\Delta \lambda_{\text {trans }}\right)$ can be connected with the width of the magnetization hysteresis loop $(\Delta M)$ by a simple formula [4]:

$$
\Delta \lambda_{\text {trans }}=\left(\frac{1-\nu}{E}\right) B_{\mathrm{e}} \Delta M,
$$

where $B_{\mathrm{e}}$ is the induction of the external magnetic field. $E$ and $\nu$ are the elastic constants - Young's modulus and Poisson's ratio, respectively. For the same sample geometries it is also possible to correlate the longitudinal strain $\left(\lambda_{\text {long }}\right)$ with the transverse one by the formula $[5,6]$ :

$$
\lambda_{\text {long }}=\left(\frac{-2 \nu}{1-\nu}\right) \lambda_{\text {trans }} .
$$

It is, however, necessary to emphasize that the stress induced in the superconducting sample by the pinning forced is not uniformly distributed in the sample volume. For some specific sample geometries this may cause changes of the sample shape and a precise analysis of the sample strain is more difficult [7].

In ceramic HTSs the widths of both magnetization and magnetostriction hysteresis loops are usually several orders of magnitude lower than in large single crystals, because in this case both magnetic moments and the strains of the samples are induced mainly by the screening currents circulating in the grains, whose diameters are usually several orders of magnitude smaller than those of the single crystals. Typical ceramic HTS sample consists of a set of superconducting grains connected by the weak links. The magnetostriction of the weakly linked medium was analyzed theoretically by Sergeenkov et al. [8], however the predictions of this theory have not been confirmed experimentally so far. Nevertheless, some of the experimental data suggest an influence of the intergrain screening currents on the magnetostriction in the range of relatively small magnetic fields [9].

Fast neutron irradiation is a well-known method of increasing the intragrain critical current density in many HTSs, by introducing in a controlled way defects acting as pinning centers. In ceramic samples, however, this method usually decreases density of the intergrain critical current [10]. The comparison of the magnetostriction of the irradiated and unirradiated HTS ceramics may be very helpful in understanding the magnetostriction mechanism in these samples. In our previous paper [4] we reported about the magnetostriction of an order of $10^{-6}$ at $4.2 \mathrm{~K}$ and $12 \mathrm{~T}$ in a ceramic sample of $\mathrm{YBa}_{2} \mathrm{Cu}_{3} \mathrm{O}_{7-\delta}$. The sample was next subjected to the fast neutron irradiation. In the present paper we compare the magnetostriction of this sample before and after irradiation. 


\section{Experimental details}

The investigated $\mathrm{YBa}_{2} \mathrm{Cu}_{3} \mathrm{O}_{7-\delta}$ ceramic sample was in a form of a slab with dimensions approximately $7.5 \times 4 \times 1.5 \mathrm{~mm}^{3}$. Magnetostriction measurements were performed by the strain gauge technique. The changes of the sample dimension were always measured in the direction parallel to the longest edge of the sample. The external magnetic field was either perpendicular to the surface of the slab (transverse magnetostriction) or parallel to the longest edge of the sample (longitudinal magnetostriction). Magnetization measurements were performed using a vibrating sample magnetometer, in which the investigated sample was placed with its longest edge parallel to the external magnetic field.

The measurements were performed both before irradiation and after irradiation of the sample by fast neutrons with the fluency of $7.55 \times 10^{17} \mathrm{n} / \mathrm{cm}^{2}$ and energy of above $0.5 \mathrm{MeV}$. Magnetostriction and magnetization hysteresis loops were registered at temperatures $4.2 \mathrm{~K}, 20 \mathrm{~K}$, and $40 \mathrm{~K}$ in an external magnetic field up to $12 \mathrm{~T}$. The external magnetic field was swept with the constant rate of about $1 \mathrm{~T} / \mathrm{min}$.

\section{Results}

Figure 1 shows the comparison of the magnetostriction (Fig. 1a transverse, Fig. 1b longitudinal) and magnetization (Fig. 1c) hysteresis loops of the investigated sample taken at $4.2 \mathrm{~K}$ before (solid line) and after (dotted line) irradiation by fast neutrons. It is clearly seen that after fast neutron irradiation the widths of both magnetostriction and magnetization hysteresis loops increased several times. A similar increase was found also at temperatures of $20 \mathrm{~K}$ and $40 \mathrm{~K}$.

In the Table we show the enhancement factors of the widths of the magnetostriction (transverse and longitudinal) and magnetization hysteresis loops in

\section{TABLE}

Enhancement factors of the widths of magnetostriction (transverse and longitudinal) and magnetization hysteresis loops at $4.2 \mathrm{~K}, 20 \mathrm{~K}$, and $40 \mathrm{~K}$ in the external magnetic field of $5 \mathrm{~T}$ and $10 \mathrm{~T}$.

\begin{tabular}{|c|c|c|c|c|c|c|}
\hline \multirow{4}{*}{$\begin{array}{c}\text { Temperature } \\
(\mathrm{K})\end{array}$} & \multicolumn{6}{|c|}{ Enhancement factor } \\
\hline & \multicolumn{3}{|c|}{ Field $5 \mathrm{~T}$} & \multicolumn{3}{|c|}{ Field $10 \mathrm{~T}$} \\
\hline & \multicolumn{2}{|c|}{ Magnetostriction } & \multirow{2}{*}{$\begin{array}{l}\text { Magneti- } \\
\text { zation }\end{array}$} & \multicolumn{2}{|c|}{ Magnetostriction } & \multirow{2}{*}{$\begin{array}{c}\text { Magneti- } \\
\text { zation }\end{array}$} \\
\hline & $\begin{array}{l}\text { Trans- } \\
\text { verse }\end{array}$ & $\begin{array}{l}\text { Longi- } \\
\text { tudinal }\end{array}$ & & $\begin{array}{l}\text { Trans- } \\
\text { verse }\end{array}$ & $\begin{array}{l}\text { Longi- } \\
\text { tudinal }\end{array}$ & \\
\hline 4.2 & 7.1 & 6.6 & 4.7 & 5.5 & 5.5 & 3.7 \\
\hline 20 & 8.2 & 8.6 & 5.6 & 4.9 & 5 & 3.3 \\
\hline 40 & 7.2 & 6.5 & 5.2 & 3.6 & 3.6 & 2.4 \\
\hline
\end{tabular}



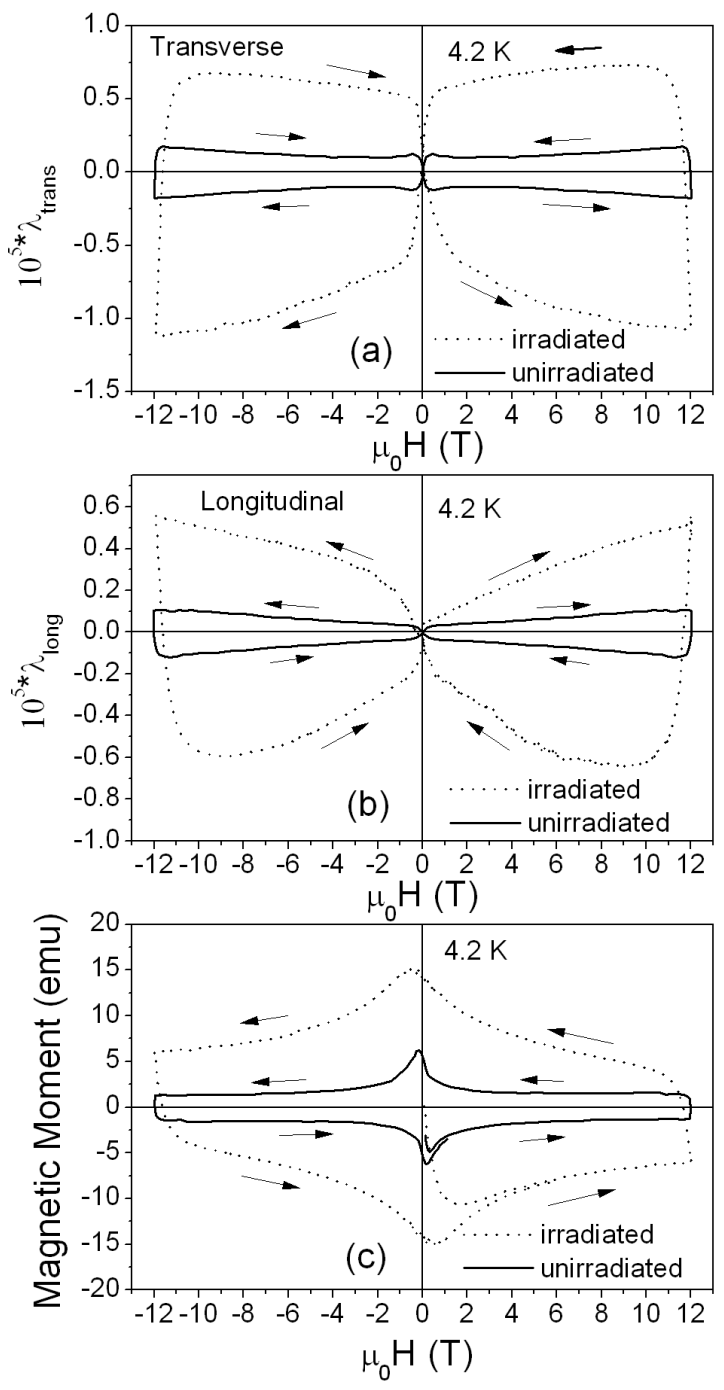

Fig. 1. Transverse (a), longitudinal (b) magnetostriction and magnetization (c) hysteresis loops of the fast neutron irradiated (dotted lines) and unirradiated (solid lines) ceramic $\mathrm{YBa}_{2} \mathrm{Cu}_{3} \mathrm{O}_{7-\delta}$ sample, taken at $4.2 \mathrm{~K}$. An increase in the magnetostriction (a,b) and of the magnetization (c) after fast neutron irradiation is shown.

the external magnetic fields of $5 \mathrm{~T}$ and $10 \mathrm{~T}$ at temperatures $4.2 \mathrm{~K}, 20 \mathrm{~K}$, and $40 \mathrm{~K}$. The numbers in the Table mean the ratios of the widths of the appropriate hysteresis loops after and before irradiation. The enhancement factors changes slightly with temperature and with the external magnetic field. At a given temperature and in a given magnetic field the enhancement factors of the transverse and longitudinal magnetostriction are almost the same and they are both slightly 
higher than an appropriate enhancement factor of magnetization. The changes of the magnetization enhancement factor with temperature and with the external magnetic field seem to follow the analogous changes of the magnetostriction enhancement factors.

\section{Discussion}

In our experiment the observed increase in the magnetostriction after fast neutron irradiation in the ceramic $\mathrm{YBa}_{2} \mathrm{Cu}_{3} \mathrm{O}_{7-\delta}$ sample can be well understood in the framework of the model of the pinning induced magnetostriction [1]. It is caused by an increase in the intragrain critical current density, which causes also an increase in the width of the magnetization hysteresis loop. According to Eq. (1) we might expect, at a given temperature and at a given external magnetic field, the same enhancement factors for both magnetostriction and magnetization hysteresis loops. In our experiment we found an increase in the magnetostriction hysteresis loop width to be slightly stronger than in the case of the hysteresis loop of magnetization. This small disagreement can be understood taking into account the approximations assumed to derive Eq. (1) - an isotropic sample with the critical current weakly dependent on magnetic field. In anisotropic HTSs probably more complex theory is necessary to obtain a better quantitative agreement with experiment. On the other hand, a good correlation between the enhancement factor of the transverse and longitudinal magnetostriction is in agreement with Eq. (2). In the framework of the model of the pinning induced magnetostriction it is also well understood that the longitudinal magnetostriction has an opposite sign as the transverse one (see the sign minus in Eq. (2) as well as arrows in Fig. 1a and $b)$.

\section{Conclusion}

The widths of both transverse and longitudinal magnetostriction hysteresis loops of ceramic $\mathrm{YBa}_{2} \mathrm{Cu}_{3} \mathrm{O}_{7-\delta}$ sample increases several times after fast neutron irradiation. The phenomenon is caused by an increase in the intragrain critical current density and can be explained in the framework of the model of the pinning induced magnetostriction.

\section{Acknowledgment}

This work was partly supported by the State Committee for Scientific Research (Poland) under contracts No. 4 T10B 02325 and No. 1 P03B 02027. 


\section{References}

[1] H. Ikuta, N. Hirota, Y. Nakayama, K. Kishio, K. Kitazawa, Phys. Rev. Lett. 70, 2166 (1993).

[2] A. Nabialek, P. Komorowski, M.U. Gutowska, M.A. Balbashov, J.N. Górecka, H. Szymczak, O.A. Mironov, Supercond. Sci. Technol. 10, 786 (1997).

[3] C.P. Bean, Phys. Rev. Lett. 8, 250 (1962).

[4] A. Nabiałek, H. Szymczak, V.A. Sirenko, A.I. D'yachenko, J. Appl. Phys. 84, 3770 (1998).

[5] T.H. Johansen, Phys. Rev. B 59, 11187 (1999).

[6] T.H. Johansen, Phys. Rev. B 60, 9690 (1999).

[7] T.H. Johansen, J. Lothe, H. Bratsberg, Phys. Rev. Lett. 80, 4757 (1998).

[8] S. Sergeenkov, M. Ausloos, Phys. Rev. B 48, 604 (1993).

[9] A. Nabiałek, H. Szymczak, K. Piotrowski, V. Chabanenko, Z. Pakieła, Physica C 321, 49 (1999).

[10] P. Przysłupski, A. Wiśniewski, S. Koleśnik, W. Dobrowolski, A. Pajạczkowska, K. Pytel, B. Pytel, Physica C 153-155, 345 (1988). 